\title{
Metabolic evaluation of children with global developmental delay
}

\author{
So-Hee Eun, MD', Si Houn Hahn, MD, PhD² \\ ${ }^{1}$ Department of Pediatrics, Korea University College of Medicine, Seoul, ${ }^{2}$ Division of Genetic Medicine, Department of Pediatrics, University of Washington School of \\ Medicine, Seattle, WA, USA
}

Global developmental delay (GDD) is a relatively common early-onset chronic neurological condition, which may have prenatal, perinatal, postnatal, or undetermined causes. Family history, physical and neurological examinations, and detailed history of environmental risk factors might suggest a specific disease. However, diagnostic laboratory tests, brain imaging, and other evidence-based evaluations are necessary in most cases to elucidate the causes. Diagnosis of GDD has recently improved because of remarkable advances in genetic technology, but this is an exhaustive and expensive evaluation that may not lead to therapeutic benefits in the majority of GDD patients. Inborn metabolic errors are one of the main targets for the treatment of GDD, although only a small proportion of GDD patients have this type of error. Nevertheless, diagnosis is often challenging because the phenotypes of many genetic or metabolic diseases often overlap, and their clinical spectra are much broader than currently known. Appropriate and cost-effective strategies including up-to-date information for the early identification of the "treatable" causes of GDD are needed for the development of well-timed therapeutic applications with the potential to improve neurodevelopmental outcomes.

Key words: Metabolism, Inborn errors, Developmental disabilities

\section{Introduction}

Global developmental delay (GDD) is defined as a significant delay in two or more domains of development, including activities of daily living as well as motor, cognitive, speech/language, and personal/social skills ${ }^{1,2)}$. The etiology of GDD is heterogeneous, and its diagnosis has been proven to be very difficult; thus, the causes of GDD are undetermined in approximately $62 \%$ of children ${ }^{3)}$. Individuals in whom genetic causes are established generally undergo very exhaustive, expensive, and often invasive diagnostic evaluations, even though the results may not change the medical or therapeutic management of the delay. Therefore, a diagnostic approach focusing on treatment has been proposed as an alternative for patients diagnosed with GDD. Inborn errors of metabolism (IEM) are uncommon causes of GDD; however, metabolic evaluations of GDD are an important step because there is an increasing availability of treatments for metabolic conditions, which may lead to better clinical outcomes. A recent study with next-generation sequencing analysis (NGS) revealed that the clinical phenotypes of many genetic or metabolic causes of GDD overlap, making diagnosis difficult and challenging. Therefore, evidence-based cost-effective strategies for the identification of treatable conditions at the early stages of GDD evaluation may be helpful in clinical practice, particularly where financial support is limited.
Corresponding author: So-Hee Eun, MD

Department of Pediatrics, Korea University Ansan Hospital, 123 Jeokgeum-ro, Danwon-gu, Ansan 425-707, Korea

Tel: +82-31-412-5096

Fax: +82-31-405-8591

E-mail: sheun@korea.ac.kr

Received: 15 December, 2014

Accepted: 4 March, 2015
Copyright (C) 2015 by The Korean Pediatric Society

This is an open-access article distributed under the terms of the Creative Commons Attribution NonCommercial License (http://creativecommons.org/ licenses/by-nc/3.0// which permits unrestricted noncommercial use, distribution, and reproduction in any medium, provided the original work is properly cited. 
Table 1. Categories and causes of global developmental delay

\begin{tabular}{ll}
\hline Category & \multicolumn{1}{c}{ Causes } \\
\hline Prenatal & Genetic \\
& Chromosomal (e.g., trisomy 21, Prader-Willi syndrome) \\
& Syndromic single gene (e.g., fragile X, Rubinstein-Taybi) \\
& Nonsyndromic single gene \\
& Metabolic (e.g., phenylketonuria, galactosemia, Smith- \\
& Lemli-Opitz syndrome) \\
& Acquired \\
& Fetal alcohol syndrome \\
& Other maternal substance abuse \\
& Nutritional (e.g., maternal phenylketonuria, iodine defici- \\
& ency) \\
& Infection (e.g., rubella, toxoplasmosis, cytomegalovirus) \\
& Stroke \\
& Unknown causes (most likely genetic but may be acquired) \\
& Clinical syndromes without genetic diagnoses \\
& Multiple congenital anomalies and mental retardation \\
& Birth asphyxia \\
Infection (herpes simplex virus encephalitis or group B Strep- \\
tococcus meningitis) \\
Stroke (embolic or hemorrhagic) \\
Very low birth weight, extreme prematurity \\
Metabolic (e.g., hypoglycemia, hyperbilirubinemia) \\
Toxins (e.g., lead) \\
Infection (e.g., Haemophilus influenzae b meningitis) \\
Stroke \\
Trauma \\
Poor nutrition \\
Poverty \\
Familial \\
Nonfamilial \\
Perinatal
\end{tabular}

\section{Causes of GDD}

GDD may be caused by prenatal, perinatal, postnatal, or undetermined causes (Table 1$)^{4}$. There are a large number of known specific causes of GDD, of which genetic or chromosomal causes constitute the largest category. Despite our considerable knowledge on the potential causes of GDD, the specific origin remains unknown in many individuals, although genetic causes have been increasingly recognized in patients with GDD. Clinical diagnostic tests using NGS have been implemented in several diagnostic reference laboratories in the United States.

\section{Approaches for children with GDD}

The initial clinical assessment of children with GDD involves the evaluation of their medical history, including prenatal, perinatal, and postnatal histories; reviewing their family history of 3 generations or more; physical and neurological examinations; and a thorough assessment of behavioral signs that may indicate a specific disease. The child's family history should be carefully obtained, while assessing whether other family members have similar or other potentially relevant neurological impairments. In addition, the mother's gestational history and pregnancy with the affected child (i.e., evidence of adverse events, toxin exposures, and intrauterine difficulties) and timing and mode of delivery (i.e., reasons for forceps application or Cesarean section) should be noted. Furthermore, the child's birth weight, Apgar scores, head circumference measurement at birth, and duration of postnatal hospital stay are important objective markers of newborn health status. Difficulties in the newborn nursery, particularly those due to possible neonatal encephalopathy (e.g., seizures, feeding difficulties prompting gavage), should be carefully documented. It is also important to obtain newborn screening (NBS) results. NBS

Table 2. Helpful diagnostic signs and symptoms in global developmental delay

\begin{tabular}{|c|c|c|c|c|}
\hline Eye abnormalities & Hearing abnormalities & Skin & Hair & Others \\
\hline Cataracts & Conduction deafness & Cafe' au lait spots & Fine hair & Hepatosplenomegaly \\
\hline Cherry-red spot & Hyperacusis & Depigmented nevi & Friable and tufted hair & Vomiting \\
\hline Chorioretinitis & Sensorineural deafness & Eczema & Loss of scalp hair & Metabolic acidosis \\
\hline Corneal ulcers & & Linear nevus & Premature gray hair & Odors \\
\hline Dislocated lenses & & Malar flush & White hair (patches) & Broad thumbs and toes \\
\hline Glaucoma & & Rash & & Abnormal fat pad \\
\hline Nystagmus & & Synophrys & & Short stature \\
\hline Photophobia & & & & Ataxia \\
\hline Retinitis pigmentosa & & & & Choreoathetosis \\
\hline \multirow[t]{3}{*}{ Vertical supranuclear gaze palsy } & & & & Dystonia \\
\hline & & & & Tremor \\
\hline & & & & $\begin{array}{l}\text { Anterior horn cell disease } \\
\text { Peripheral neuropathy }\end{array}$ \\
\hline
\end{tabular}


panels that use tandem mass spectrometry dramatically improve early diagnosis and outcomes with appropriate treatments, but current NBS tests target only a minority of treatable IEM. Parental recall of motor skill development in the first year of life and speech/language milestones is important. The potential loss or regression of previously acquired developmental skills should be particularly noted because this indicates the need for a more urgent etiologic evaluation and follow-up. Current skill levels in various developmental domains together with the degree of independence in activities of daily life, scholastic history, and coexisting medical problems should be noted.

Physical examination should assess possible dysmorphology, hepatosplenomegaly, and cutaneous markers of neurocutaneous syndromes as well as the presence of dermal sinuses, hair tufts, or other subtle signs of spinal dysraphism in the sacral region. Furthermore, height, weight, and head circumference should be measured. Additional signs and symptoms can help to identify the causes of GDD (Table 2$)^{4)}$.

After clinical evaluation, appropriate diagnostic laboratory tests, brain imaging, and other evidence-based evaluations are applied for establishing diagnosis and for care planning ${ }^{5)}$. With remarkable progress in the field of genetics over the last several years, chromosomal microarrays are now important first-line clinical diagnostic tests for children with GDD of unknown causes. Moreover, a renewed emphasis on the identification of "treatable" causes of GDD recommends screening for IEM in

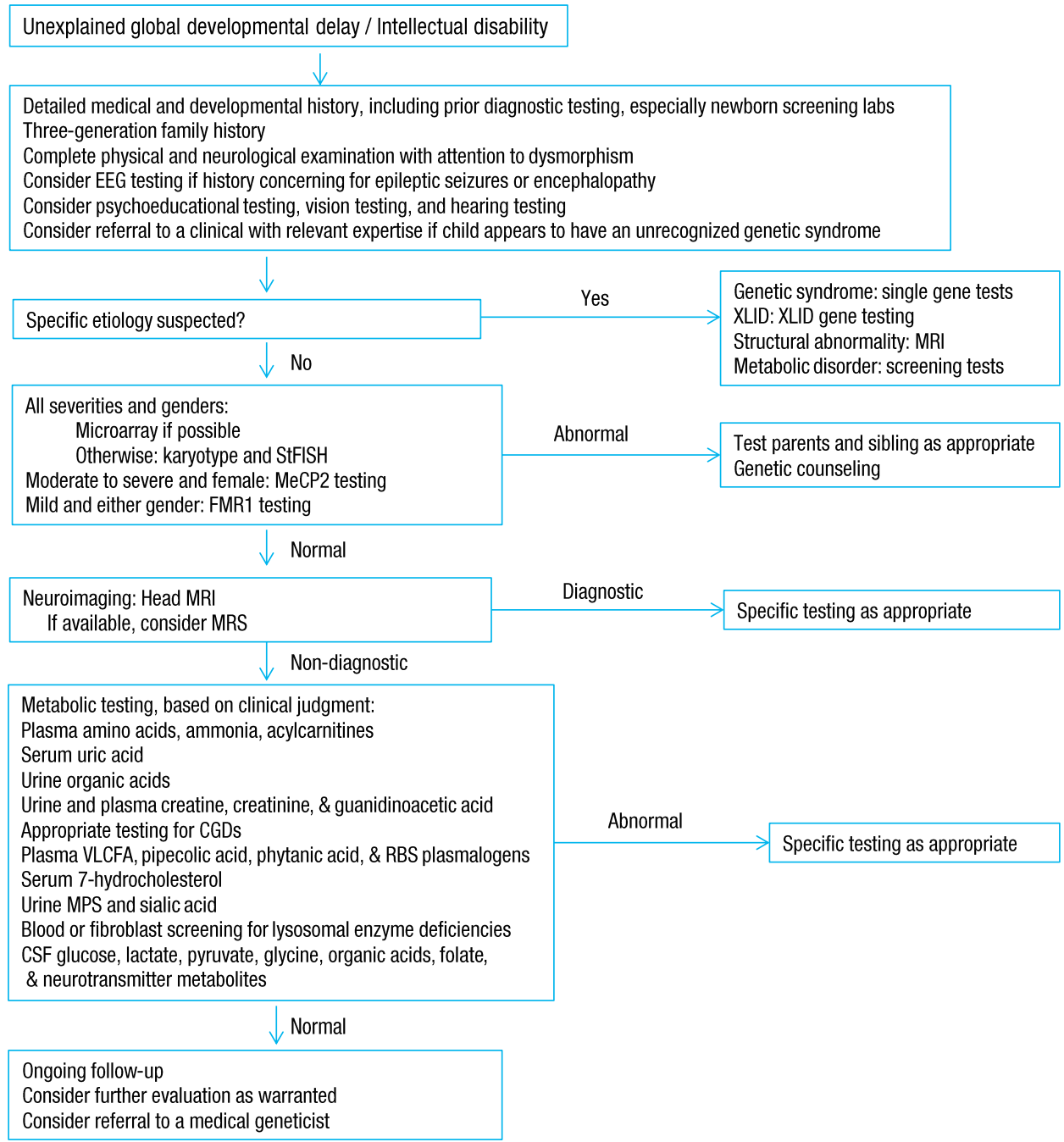

Fig. 1. Diagnostic strategy for the evaluation of children with unexplained global developmental delay or intellectual disability. EEG, electroencephalogram; XLID, X-linked intellectual disability; StFISH, subtelomeric fluorescence in situ hybridization; MeCP2, methyl $\mathrm{CpG}$ binding protein 2; FMR1, fragile $\mathrm{X}$ mental retardation 1; MRI, magnetic resonance imaging; MRS, magnetic resonance spectroscopy; CGD, congenital disorder of glycosylation; VLCFA, very long chain fatty acids; MPS, mucopolysaccharidosis; CSF, cerebrospinal fluid. Adapted from Sherr et al. Swaiman's pediatric neurology. 5th ed. 2012:562 ${ }^{4}$. 
children with GDD of unknown causes ${ }^{4-6)}$. The practice parameters and evidence report for GDD or intellectual disability published by the American Academy of Neurology provide a framework of recommendations for evaluation (Fig. 1). These include a combination of broad screening tests and disease-specific evaluations based on a heightened pretest probability (>1\%), while considering the identified clinical features ${ }^{4)}$.

\section{Characteristics of developmental delay caused by IEM}

Developmental delay is a major symptom of various IEM that appear at a young age. Therefore, understanding the general characteristics of developmental delay caused by IEM is an important step for better identification and appropriate follow-up with metabolic workup.

First, developmental delay caused by IEM is usually global, which implies that it affects, to some extent, all domains of development. Second, behavioral problems such as severe irritability, impulsivity, aggressiveness, and hyperactivity are common. Typical examples are Krabbe disease (globoid cell leukodystrophy), Sanfilippo disease (mucopolysaccharidosis type
III [MPS III]), and Hunter disease (MPS II). Motor automatisms and stereotypic behaviors are also common in these disorders. Selfmutilation, such as compulsive chewing of the thumb and fingers, sometimes resulting in severe laceration of the lips and traumatic amputation of the fingers, is a prominent symptom in boys with Lesch-Nyhan syndrome. Third, delayed development is usually progressive in children with IEM. Developmentally delayed children with IEM may show a period of normal development, followed by a loss of developmental milestones. The progression may be subtle at first, but the gap between children with GDD and healthy children becomes wider with time. Finally, delayed development in children with IEM is usually associated with other objective neurological dysfunctions such as abnormal muscle tone, problems in specific senses, seizures, pyramidal tract signs, and extrapyramidal or cranial nerve deficits.

Age at onset and clinical course often provide important clues to the metabolic nature of the disease, and the involvement of other organs is frequent. The major affected areas outside the central nervous system include organs such as the liver and spleen, as well as the muscles, bones, skin, and connective tissues (Fig. 2). An initial evaluation for children with undifferentiated developmental delay includes thorough developmental assessment and neurological examination, brain imaging, electrophysiological

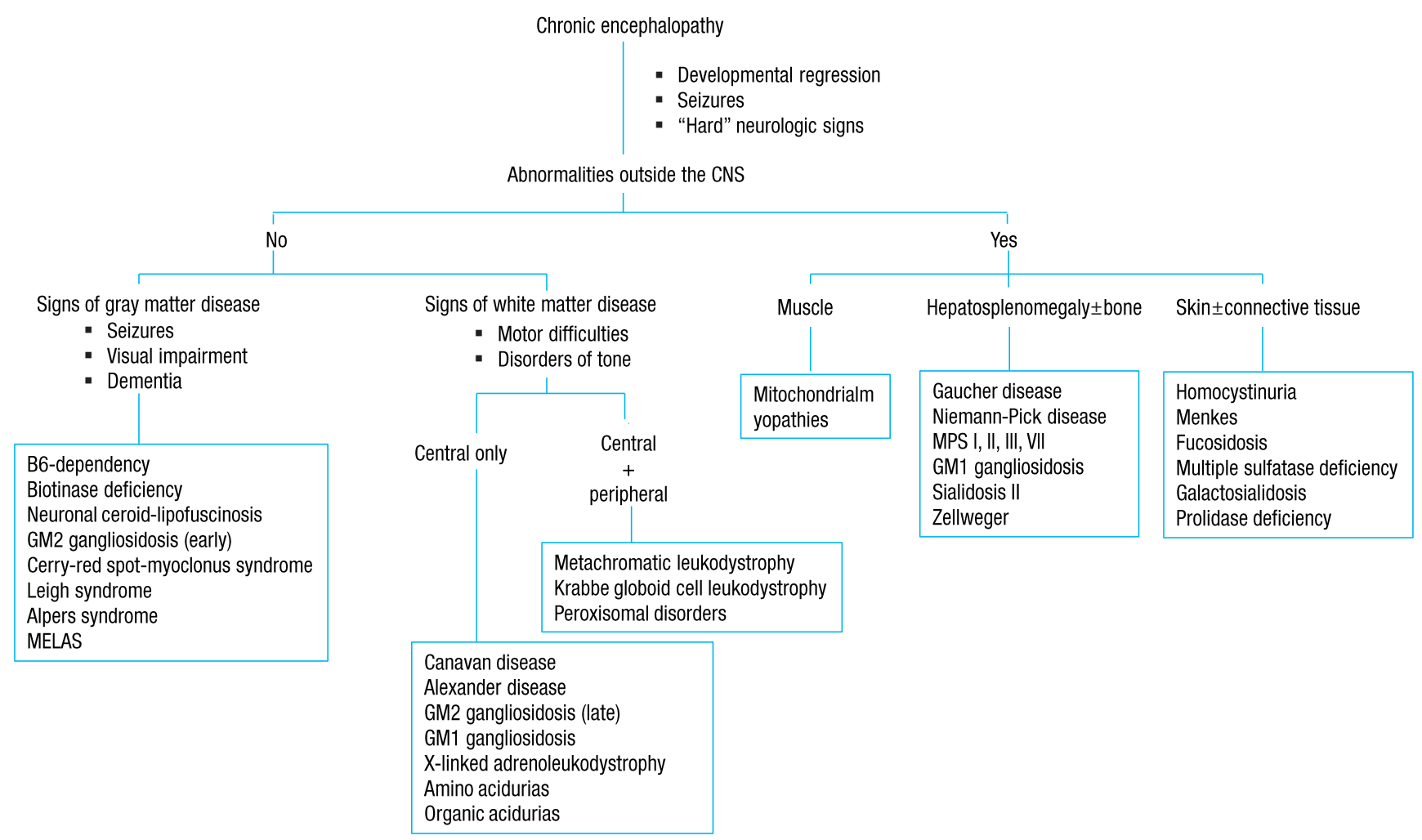

Fig. 2. An approach to inherited metabolic diseases with chronic encephalopathy. CNS, central nervous system; MELAS, mitochondrial encephalomyopathy, lactic acidosis, and stroke-like episodes syndrome; MPS, mucopolysaccharidosis. Adapted from Clarke. A clinical guide to inherited metabolic diseases. 2006:33'. 
studies (auditory brainstem responses, visual evoked potentials, somatosensory evoked potentials, nerve conduction studies, electromyogram), radiographs of the hands, chest, and lateral view of the spine for evidence of dysostosis multiplex, plasma amino acid analysis, urinary amino acid analysis, urinary organic acid analysis, even in the absence of overt metabolic acidosis, plasma ammonium, preferably two hours after a normal meal of protein-containing food, plasma lactate, urinary MPS screening, and urinary oligosaccharide screening test. It also includes tests for determining the extent and degree of neurological damage, ensuring that the early stages of some treatable IEM are not missed, and establishing a baseline for monitoring the natural history of the disease ${ }^{7)}$.

\section{Diagnostic recommendations for the identification of treatable causes of GDD}

If a diagnosis is unclear after a clinical assessment, a stepwise evaluation process is undertaken. At this stage, specific metabolic testing should be considered, including total serum homocysteine, acylcarnitine profile, amino acids, organic acids in urine, glycosaminoglycans, oligosaccharides, purines and pyrimidines, and guanidinoacetate-to-creatine ratio. Routine metabolic testing of children with GDD had a $0.8 \%-2.5 \%$ rate of diagnosis of $\mathrm{IEM}^{5,8,9)}$, but a detailed metabolic reassessment identified the causative IEM in up to $14 \%$ of the patients previously classified as having GDD of an unknown cause ${ }^{10,11}$. These studies have raised the concern that treatable diagnoses may be missed if we focus too heavily on current practice parameters ${ }^{12,13)}$.

In 2013, an updated systematic literature review identified 89 IEM, which present with GDD as a prominent feature and are amenable to causal therapy ${ }^{14)}$. Most IEM are associated with additional neurological and/or nonneurological features. Neurological features include ataxia, behavioral disturbances, dementia, dystonia, encephalopathic crisis, epilepsy, hearing loss, hypotonia, myopathy, abnormalities in brain imaging (basal ganglia, cerebellum, cerebrum, white matter, and mixed), neuropathy, ocular movement abnormalities, psychiatric disturbances, sensorineural hearing loss, spasticity, stroke, or vision loss. Most IEM are associated with at least one additional prominent neurological feature, such as epilepsy and various types and degrees of movement disorders (e.g., spasticity, dyskinesia, and ataxia). However, many cases of IEM present with developmental delay as the sole feature for a considerable amount of time before manifestation of the full phenotype (e.g., disorders of creatine synthesis and transport). The nonneurological features affect the following anatomical/organ systems: bones and joints; skin and blood tissues; gastrointestinal, endocrine, and immune systems; organs including the eyes, liver, heart, and kidneys; and anatomical features including facial dysmorphism, growth, and stature.

These 89 IEM include disorders of specific molecules such as amino acids $(\mathrm{n}=12)$, cholesterol and bile acids $(\mathrm{n}=3)$, vitamins/ cofactors $(n=10)$; creatine $(n=3)$, fatty aldehydes $(n=1)$, purines and pyrimidines $(n=3)$; metals $(n=5)$, organic acids $(n=19)$; disorders that affect cellular organelles such as lysosomes $(n=12)$, mitochondria $(n=2)$, and peroxisomes $(n=1)$; anomalies

\begin{tabular}{|c|c|}
\hline \multicolumn{2}{|c|}{$1^{\text {st }}$ tier: non-targeted screening } \\
\hline $\begin{array}{l}\text { Blood } \\
\text { Ammonia, lactate } \\
\text { Plasma amino acids } \\
\text { Total homocysteine } \\
\text { Acylcarnitine profile } \\
\text { Copper, ceruloplasmin }\end{array}$ & $\begin{array}{l}\text { Urine } \\
\text { Organic acids } \\
\text { Purines and pyrimidines } \\
\text { Creatine metabolites } \\
\text { Oligosaccharides } \\
\text { Glycosaminoglycans }\end{array}$ \\
\hline \multicolumn{2}{|l|}{$2^{\text {nd }}$ tier: targeted testing } \\
\hline \multicolumn{2}{|c|}{$\begin{array}{l}\text { Consider the following biochemical/molecular analyses: } \\
\text { Whole blood manganese } \\
\text { Plasma cholesterol } \\
\text { Plasma 7-dehydroxy-cholesterol:cholesterol ratio } \\
\text { Plasma pipecolic acid \& urine amino adipic semialdehyde } \\
\text { Plasma very long chain fatty acids } \\
\text { Plasma vitamin } B_{12} \text { and folate } \\
\text { Serum \& cerebrospinal fluid lactate:pyruvate ratio } \\
\text { Enzyme activities (leukocytes): arylsulfatase A, biotinidase, glucocerebrosidase, fatty aldehyde dehydrogenase } \\
\text { Urine deoxypyridinoline } \\
\text { CSF amino acids } \\
\text { CSF neurotransmitters } \\
\text { CSF: plasma glucose ratio } \\
\text { Coenzyme Q measurement fibroblasts } \\
\text { Molecular: CA5A, NPC1, NPC2, SC4MOL, SLC18A2, SLC19A3, SLC30A10, SLC52A2, SLC52A3, PDHA1, DLAT, PDHX, SPR, TH }\end{array}$} \\
\hline
\end{tabular}

Fig. 3. A two-tiered diagnostic strategy for treatable inborn errors of metabolism in intellectual developmental disorder. Adapted from van Karnebeek et al. Mol Genet Metab 2014;111:428-38 ${ }^{14)}$. 
in glucose homeostasis and transport $(n=2)$, the urea cycle $(n=8)$, neurotransmission $(n=8)$, and hyperhomocysteinemia $(n=7)$.

Fifty-four of the 89 disorders (60\%) are identified by metabolic screening tests in blood (plasma amino acids, homocysteine, copper, and ceruloplasmin) and urine (creatine metabolites, glycosaminoglycans, oligosaccharides, organic acids, and pyrimidines). For the remaining 35 disorders (40\%), specific tests are required, including primary molecular analysis. First-tier testing includes group metabolic tests in urine and blood, which should be performed in every patient with GDD of unknown cause. Based on the differential diagnosis generated by the patient's signs and symptoms, a second-tier test is prescribed individually using a low threshold. Selective metabolic testing should be performed if there are suggestive clinical findings, including lethargy, cyclic vomiting, early seizures, dysmorphic features, and developmental disabilities (Fig. 3) ${ }^{14}$.

\section{Conclusions}

Recent advances in genetic technology provide the possibility of improving diagnosis, and ultimately the outcomes, in patients with GDD. This could allow early diagnosis and the use of available treatments for the specific causes of GDD. Neurological pathologies are the most prominent clinical symptoms associated with many types of GDD, and, in particular, with IEM. However, general neurological issues are common, and deciding who should be evaluated and the type of testing needed is often challenging. Although metabolic disorders represent a small and extremely heterogeneous proportion of GDD cases, early diagnosis of metabolic disorders and proper therapeutic interventions may significantly improve the developmental outcomes in some of these patients. Therefore, the clinical application of innovative evidence-based evaluations for appropriate metabolic and genetic testing of GDD of unknown causes should be strongly considered.

\section{Conflict of interest}

No potential conflict of interest relevant to this article was reported.

\section{References}

1. Shevell M, Majnemer A, Platt RW, Webster R, Birnbaum R. Developmental and functional outcomes in children with global developmental delay or developmental language impairment. Dev Med Child Neurol 2005;47:678-83.

2. Shevell M, Ashwal S, Donley D, Flint J, Gingold M, Hirtz D, et al. Practice parameter: evaluation of the child with global developmental delay: report of the Quality Standards Subcommittee of the American Academy of Neurology and The Practice Committee of the Child Neurology Society. Neurology 2003;60:367-80.

3. Jimenez-Gomez A, Standridge SM. A refined approach to evaluating global developmental delay for the international medical community. Pediatr Neurol 2014;51:198-206.

4. Sherr EH, Shevell MI. Global developmental delay and mental retardation/intellectual disability. In: Swaiman KF, Ashwal S. Ferriero DM, Schor NF. Swaiman's pediatric neurology. 5th ed. Elsevier Saunders, 2012:554-74.

5. Moeschler JB, Shevell M; Committee on Genetics. Comprehensive evaluation of the child with intellectual disability or global developmental delays. Pediatrics 2014;134:e903-18.

6. van Karnebeek CD, Jansweijer MC, Leenders AG, Offringa M, Hennekam RC. Diagnostic investigations in individuals with mental retardation: a systematic literature review of their usefulness. Eur J Hum Genet 2005;13:6-25.

7. Clarke JT. A clinical guide to inherited metabolic diseases. Cambridge: Cambridge University Press, 2006.

8. van Karnebeek CD, Scheper FY, Abeling NG, Alders M, Barth PG, Hoovers JM, et al. Etiology of mental retardation in children referred to a tertiary care center: a prospective study. Am J Ment Retard 2005;110:253-67.

9. Sempere A, Arias A, Farre G, Garcia-Villoria J, Rodriguez-Pombo P, Desviat LR, et al. Study of inborn errors of metabolism in urine from patients with unexplained mental retardation. J Inherit Metab Dis 2010;33:1-7.

10. Engbers HM, Berger R, van Hasselt P, de Koning T, de Sain-van der Velden MG, Kroes HY, et al. Yield of additional metabolic studies in neurodevelopmental disorders. Ann Neurol 2008;64:212-7.

11. Papavasiliou AS, Bazigou H, Paraskevoulakos E, Kotsalis C. Neurometabolic testing in developmental delay. J Child Neurol 2000;15:620-2.

12. Mueller S, Sherr EH. The importance of metabolic testing in the evaluation of intellectual disability. Ann Neurol 2008;64:113-4.

13. van Karnebeek CD, Stockler S. Treatable inborn errors of metabolism causing intellectual disability: a systematic literature review. Mol Genet Metab 2012;105:368-81.

14. van Karnebeek CD, Shevell M, Zschocke J, Moeschler JB, Stockler S. The metabolic evaluation of the child with an intellectual developmental disorder: diagnostic algorithm for identification of treatable causes and new digital resource. Mol Genet Metab 2014; 11:428-38. 